\title{
Development of a Computerized Model of Performance-Based Measurement to Facilitate and Evaluate New Graduate Nurses' Clinical Competence
}

\author{
Shwu-Ru Liou ${ }^{1} \quad *$ Ching-Yu Cheng ${ }^{1} \quad$ Hsiu-Chen Liu ${ }^{1}$ Shu-Ling, Tsai ${ }^{1}$ \\ ${ }^{1}$ Chang Gung University of Science and Technology at Chia-Yi Campus
}

\begin{abstract}
New graduate nurses are not adequately prepared for performing as expected by the clinical settings to meet the growing demands of complex healthcare environment. The purpose of the study was to develop a Computerized Model of Performance-Based Measurement (CMPBM) to improve and evaluate clinical competence of pre-graduate nursing students and new nurses to better prepare and facilitate their transition to clinical work environment. The top 10 high-risk, high-volume medical and surgical scenarios were selected to build into the CMPBM. The Clinical Reasoning Model was used as a guideline to develop scenarios. Eight construction principles were applied for developing the CMPBM.
\end{abstract}

Keywords: performance-based measurement, clinical competence, new graduate nurses

\section{Introduction}

Nursing shortage has become a global issue as well as high nurse turnover rate. Nevertheless, registered nurse turnover is costly to organizations in terms of quality of patient care and both implicit and explicit financial costs [1]. Hiring new graduate nurses may be a significant way and urgent solution to relieve nurse shortage problem. Unfortunately, new graduate nurses are not adequately prepared for performing as expected by the clinical settings to meet the growing demands of today's complex healthcare environment [2]. In a study, only $10.4 \%$ of the hospital nurse executives agreed that new graduate nurses are fully prepared to provide safe and effective care in the hospital setting [3]. Nurse administrators pointed out that new graduate nurses have difficulty in critically thinking on managing patient situations with quick and appropriate decision making [2].

In response to the clinical competence issue among new graduate nurses, researchers propose to design and develop a performance-based evaluate system to train and assess nursing students and new nurses' critical thinking ability, which is necessary and should be adequate for practice in their future workplace $[4,5]$. The purpose of the study was to develop a Computerized Model of Performance-Based Measurement (CMPBM) to improve and evaluate the competence of nurses pre-graduate nursing students and new graduate to better prepare them and facilitate their smooth transition to clinical environment.

\section{Methods}

\subsection{Applying the Model of Clinical Reasoning for Developing the CMPBM}

This study focused on the clinical competencies of assessment and intervention, communication, critical thinking, human caring and relationships, and knowledge integration skills [6]. To achieve the goals of competencies in the definition, we designed the CMPBM based on literature reviews including two major dimensions: critical thinking and interpersonal skills. The definition of critical thinking that is interchangeable with clinical reasoning is characterized as a problem-solving process, whereby information is gathered and interpreted, hypotheses generated and decisions made [7]. Similarly, the clinical reasoning is described as gathering information, interpreting information, deciding actions and reviewing the outcomes [8].

To construct the CMPBM, we did an extensive literature review and research into how expert nurses think. We then chose and applied the Clinical Reasoning Model (CRM, Figure 1) developed by Hoffman and colleagues to build and format each step in the CMPBM $[9,10]$.

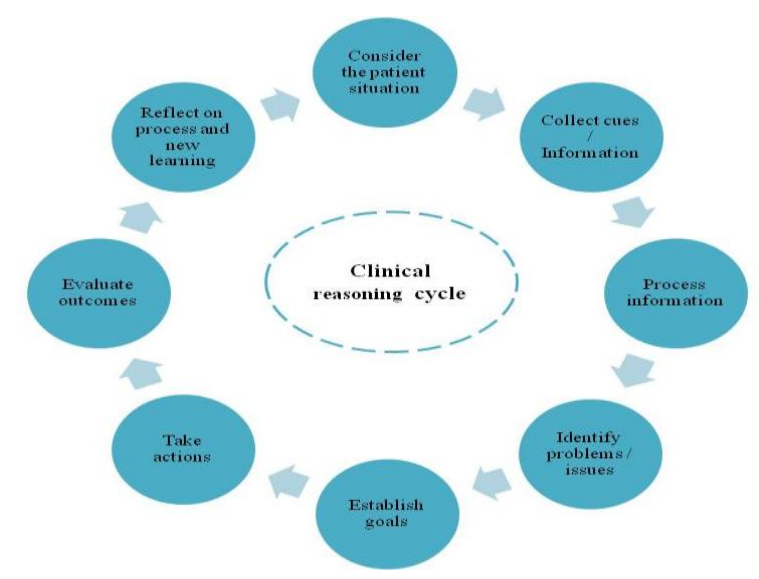

Figure. 1: The Clinical Reasoning Model applied for the development of the CMPBM.

The model proposes that clinical reasoning is a process by which nurses and other clinicians collect cues, process the information, come to an 
understanding of a patient problem, plan and implement interventions, evaluate outcomes, and reflect on and learn from the process. The steps in the model involve higher order thinking skills such as gathering information, recalling knowledge, reviewing, synthesizing, and interpreting information, and making inferences of patient conditions [10].

\subsection{Developing Scenarios Based on Clinical Cases and Situations}

The Clinical Reasoning Model (CRM) contains eight steps (Figure 1) and was used as a guideline to establish scenarios to build into the CMPBM to test and evaluate students' or nurses' abilities in clinical critical thinking and clinical competence. These eight steps include: consider the patient situation, collect cues/information, process information, identify problems/issues, establish goal(s), take action, evaluate outcomes, and reflect on process and new learning.

According to literatures, the top 6 high-risk, high volume medical and surgical situations (e.g. liver cirrhosis, diabetes mellitus, cerebrovascular accident) were selected to develop scenarios for the measurement of competence. Each scenario includes a series of patient situations that induce students to apply step-by-step clinical reasoning thinking and finally to solve patients' issues through critical thinking. All the scenarios were based on real patient situations to make all the learning and skill acquisition among participants be tied to authentic contexts.

According to the Clinical Reasoning Model, in the first step, a situation providing information including contextual facts about the patient was described. In the second step, participants collected and reviewed patient information provided in the scenario with previous obtained knowledge and experiences. In the third step, a situation was given for the participants to apply reasoning skills such as discriminating, relating, interpreting, and predicting to process the information collected. The fourth step provided a situation that was for the participants to identify patient issues. The fifth step involved setting goals of care for the patient issues. The sixth step required the participants to provide nursing actions to the case. The seventh step involves evaluation of the cares. The last step includes reflection-on-action by students themselves.

The situations in a scenario case sequenced to reflect a patient's changing condition, either improvement or deterioration. To promote interactivity, different types of question were designed: multiple choice, click and drag, and open-ended questions. In addition, components in the dimension of interpersonal skills such as conflict resolution, customer relations and satisfaction, or team building were designed into the program with clinical scenarios for participants to learn how to manage the complicate interrelationships among healthcare colleagues and patients.

\subsection{Construction Principles of the CMPBM}

The CMPBM is designed to encourage the development of specific knowledge and critical thinking skills. The main principle for the development of the CMPBM is providing opportunities for participants to construct knowledge for themselves through interacting, thinking and making decision of the cases that are situated, experiential and authentic [10]. Eight principles were developed to guide the design of the CMPBM (Figure 2, 3).

Principle 1. Participants are allowed to make mistakes on answering questions developed specifically for each computerized scenarios. By doing so can reinforce learning and allow construction of experiences and knowledge.

Principle 2. An interactive computerized platform (ubuntu 10.04) and programs such as php 5.3.2, mysql 5.1.41, and LimeSurvey 2.0 are used to develop the CMPBM. The CMPBM was constructed to allow interactivity. All scenarios were designed according to steps of the CRM. Patient situations and competences that were intended to achieve in each scenario were sequenced to reflect a patient's changing conditions, either improvement or deterioration. Before beginning each scenario tests, participants were required to read through and answer questions on thinking like an experienced nurse" and this was described as an introduction to the scenarios.

Principle 3. Situations of each case were established follow each step of the Clinical Reasoning Model. Several questions followed a description of patient situations. The participants needed to answer al questions for each situation correctly before they could proceed to the next stage of the scenario.

Principle 4. Each multiple-choice question in situations had at least four answers for participants to make decision and choose. Different score was given based on different sequence of answers that were chosen. For example, when the question is answered correctly at the first time, the system automatically scored 5 points; at the second time, 3 points were given; at the third time, 2 points were scored; and at the fourth time or more, 1 point was given. The open-ended questions were evaluated by two experienced faculties after their interrater reliability on scoring was established.

Principle 5. The computer system was designed to automatically calculate and store the total score that each student obtained in every test. Furthermore, the system was able to calculate and categorize the score earned by the participants on each step of the CRM. These individual scores could help nurse educators to understand which ability the participants needed to be reinforced.

Principle 6. All the questions in each situation were designed to couple with immediate, evidence-based feedback on both correct and incorrect answers. Researchers propose that online learning that incorporates immediate, corrective feedback is critical to learning as it enhances learners' active participation and encourages motivation to finish [11].

Principle 7. The computerized scenarios are formatted to help participants achieve competence by answering all questions correctly. Therefore, all 
scenarios and all questions were computerized in the way that allowed the participants redid the scenarios as many times as needed.

Principle 8. The participants were able to work on the patient scenarios following the designed sequential steps and at their own pace. This method allowed the participants to move forward the increasing complex operations and also asked the participants to make decisions at a critical junction in the scenarios that enabled the participants to construct their own learning while proceeding smoothly from one step to the next [10].

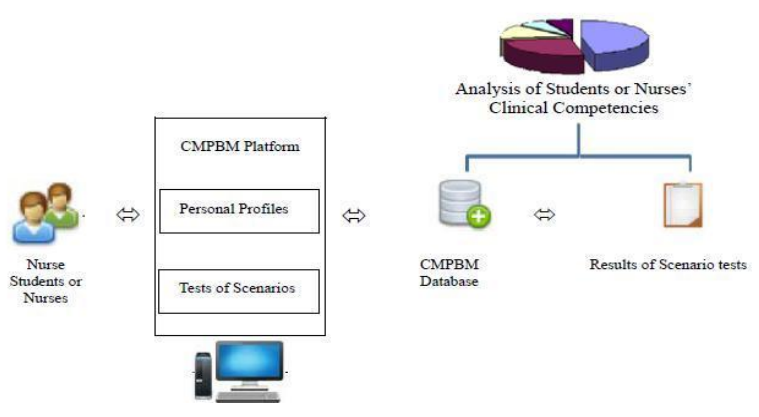

Fig. 2: The computerized Model of Performance-Based Measurement System.

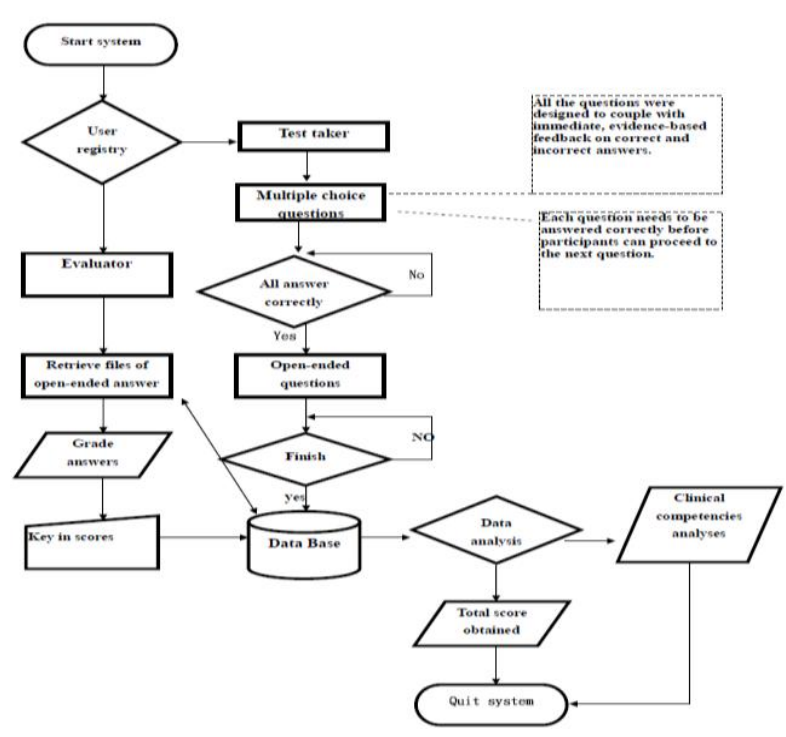

Fig. 3: Process of Computerized Model of Performance-Based Measurement.

\subsection{Conclusion}

Traditionally, nurse educators prepare students to demonstrate specific knowledge, skills, and behaviors in the context of regulatory requirements, faculty perception of practice needs, and limited credit hours for learning. The resulting level of preparation may or may not be enough and satisfactory for nurse graduates in practice. It is important that nursing pre-graduates' perceptions of their knowledge and skills at schools and actual provisions of care in clinical settings be similar because gaps between virtual perception and performance in the real world could lead to errors and patient safety issues.

Therefore, in this study, we attempted to establish an interactive computerized platform designed with scenarios based on real situations in the clinical settings for nurse students and new graduate nurses to practice as well as to measure their clinical competencies that can help them to bridge the gape between student and the clinical nurse roles. To construct the CMPBM, three main tasks were designed and implemented: applying the Clinical Reasoning Model for the development of the CMPBM, developing scenarios based on clinical situations, and constructing the CMPBM based on eight principles. Students or new graduate nurses obtained abilities of identifying changes in patient conditions, providing interventions independently, prioritizing managements, and communicating with team effectively. The next step of this study is to collect more data from nurse students in different nursing programs and new nurses to evaluate the results of using this system.

We acknowledge the National Science Council (NSC 101-2511-S-255-002-MY2) in Taiwan for funding this study. Correspondence to Ching-Yu Cheng.

\section{References}

[1] C.B. Jones, "Revisiting nurse turnover costs: Adjusting for inflation," Journal of Nursing Administration, 38 (2008) 11-18.

[2] P. Burns, and E. Poster, Competency development in new registered nurse graduates: Closing the gap between education and practice," The Journal of Continuing Education in Nursing 39 (2008) 67-73.

[3] S. Berkow, K. Virkstis, J. Stewart, and L. Conway, Assessing new graduate nurse performance. Journal of Nursing Administration 38 (2008) 468-474.

[4] L.J. Fero, C.M. Witsberger, S.W. Wesmiller, T.G. Zullo, and L.A. Hoffman, Critical thinking ability of new graduate and experienced nurses. Journal of Advanced Nursing 65 (2009) 139-148.

[5] S. Sportsman, Competency education and validation in the United States: What should nurses know? Nursing Forum 45 (2010) 140-149.

[6] C.B. Lenburg, C. Klein, V. Abdur-Rahman, T. Spencer, and S. Boyer, The COPA model: A comprehensive framework designed to promote quality care and competence for patient safety. Nursing Education Perspectives 30 (2009) 312-317.

[7] D.A. Bryce, N.J. King, C.F. Graebner, J.H. Myers, Evaluation of a medical diagnostic reasoning programme (DxR): Exploring student perceptions and addressing faculty concerns. Journal of Interactive Media in Education 98 (1998) 1-35.

[8] C.A. Tanner, Thinking like a nurse: A research-based model of clinical judgment in nursing. The Journal of Nursing Education 45 (2006) 204-211.

[9] T. Levett-Jones, K. Hoffman, J. Dempsey, S. Jeong, D. Noble, C. Norton, J. Roche, and N. Hickey, The five rights of clinical reasoning: An educational model to enhance nursing students' ability to 
identify and manage clinically at risk patients. Nurse Education Today 30 (2010) 515-520.

[10] K. Hoffman, J. Dempsey, T. Levett-Jones, D. Noble, N. Hickey, S. Jeong, S. Hunter, and C. Norton, The design and implementation of an interactive computerized decision support framework (ICDSF) as a strategy to improve nursing students' clinical reasoning skills. Nurse Education Today 31 (2010) 587-594.

[11] K.L. Rush, C.E. Dyches, S. Waldrop, and A. Davis Critical thinking among RN to BSN distance students participating in human patient simulation. Journal of Nursing Education 47 (2008) 501-507. 